\title{
marges Marges
}

revue d'art contemporain Revue d'art contemporain

\section{$23 \mid 2016$}

\section{Globalismes}

\section{Éditorial}

Jérôme Glicenstein

\section{OpenEdition}

\section{Journals}

Édition électronique

URL : http://journals.openedition.org/marges/1187

DOI : 10.4000/marges. 1187

ISSN : 2416-8742

\section{Éditeur}

Presses universitaires de Vincennes

\section{Édition imprimée}

Date de publication : 20 octobre 2016

Pagination : 5-8

ISBN : 978-2-84292562-8

ISSN : 1767-7114

Référence électronique

Jérôme Glicenstein, «Éditorial », Marges [En ligne], 23 | 2016, mis en ligne le 20 octobre 2016, consulté le 25 septembre 2020. URL : http://journals.openedition.org/marges/1187 ; DOI : https://doi.org/ $10.4000 /$ marges. 1187 


\section{Éditorial}

Le constat d'un «Art global » offre, depuis la fin des années 1980, une importante source de réflexions aux théoriciens et historiens de l'art, ainsi qu'aux commissaires d'exposition et professionnels des musées. Selon le spécialiste des Global Studies, Hans Belting, l'art contemporain peut être appréhendé aujourd'hui comme synonyme de global art; le " globalisme " étant selon lui l'antithèse de l'universalisme, dans la mesure où il décentre une vision du monde unifiée, tout en s'orientant vers des modernités multiples. Un grand nombre de colloques, séminaires, publications, programmes de recherche, accrochages de musées et d'expositions, font écho à ces questions. La littérature produite sous l'étiquette de Global Studies met généralement en avant deux approches: la première relie l'art contemporain à l'évolution du musée, des biennales, des foires, etc., à la lumière de la globalisation économique; la deuxième dénonce les inégalités dans les modalités d'accès au monde de l'art contemporain et considère que le processus d'institutionnalisation ne fonctionne pas de la même manière pour tous.

La première approche traduit assez bien les évolutions récentes des institutions artistiques et notamment la volonté de plus en plus affichée de repenser la programmation des musées ou leur politique d'acquisition en incluant des zones géographiques auparavant marginalisées. La deuxième approche souhaite quant à elle élaborer des paradigmes critiques et historiographiques capables de prendre en compte les apports des études postcoloniales, des études culturelles et, plus récemment, de la géographie critique. Au cours des vingt dernières années, un grand nombre de questions et de catégories d'interprétation nouvelles ont ainsi été mobilisées, introduisant des 
notions inédites dans l'historiographie de l'art, telles que métissage, exclusion, décentrement, hybridation, etc. En empruntant des termes et approches autres que ceux de l'histoire de l'art, ces nouvelles lectures promettent une réorganisation des discours sur l'art qui prenne en compte des récits multiples et fragmentaires, réfractaires aux catégories du canon moderniste occidental.

Vingt ans de débats sur l'émergence d'un monde global ont néanmoins principalement conduit à la production de nouvelles " étiquettes ", lesquelles se réfèrent à la géographie (art africain, art latino-américain...) au médium (photographie, performance) et aux courants canoniques occidentaux (surréalisme, figuration/abstraction, conceptualisme...). Ces étiquettes semblent remettre au goût du jour des catégories éprouvées, tout en réduisant la production des pays extra-occidentaux à des concepts facilement exploitables, voire vendables. L'Art global serait-il en train de se transformer en un nouveau canon contemporain ? Quelles possibilités concrètes y a-t-il de refuser un tel canon et de créer de nouveaux outils pour penser de nouvelles perspectives pour l'art de notre temps? Si ce numéro s'intéresse à la question de l'Art global, ce n'est pas du point de vue d'une unification des pratiques artistiques à l'échelle mondiale, mais bien plus en prenant en compte les effets de la globalisation sur des pratiques locales.

La première partie s'intéresse à l'art brésilien; un cas paradigmatique sans doute en ce que la scène artistique de ce pays a vu cohabiter à la fois des pratiques modernistes occidentalisées, des pratiques visant à définir une modernité spécifiquement brésilienne et d'autres types de positionnements encore, plus difficiles à qualifier, entre rituels d'origine africaine, art religieux et traditions populaires. La question de la définition d'une spécificité artistique brésilienne est au cœur de l'article de Vinicius Spricigo. Son étude se centre sur le réinvestissement contemporain de la notion d'anthropophagisme: une notion propre à la modernité des années 1920 (Oswaldo de Andrade) et qui a été utilisée à nouveau dans les années 1990 pour qualifier la XXIVe biennale de São Paulo. En un sens, il s'agissait bien là de confronter la réalité de la scène brésilienne aux représentations dont elle fait l'objet à l'extérieur du pays. 
Camila Bechelany traite d'une situation presque opposée: la présentation de la modernité brésilienne à l'étranger, au travers de l'exposition "Modernidade, art brésilien du $20^{\mathrm{e}}$ siècle » au Musée d'art moderne de la ville de Paris en 1987. Cette exposition, ainsi que l'important catalogue qui l'accompagnait, avait en partie été produite par des institutions brésiliennes. Elle avait pour ambition de donner à voir une autre image de l'histoire de l'art au Brésil, tout en présentant une image différente de l'idée même de modernité.

Le texte de Cecilia Braschi s'intéresse enfin à des questions proches de celles des deux articles précédents, mais dans une perspective plus historique, observant la manière dont l'architecture brésilienne des années 1940-1960 a été perçue hors de ce pays dans des revues européennes et nord-américaines. À lire ces revues, la modernité occidentale semble en effet se refléter à distance dans les productions sud-américaines; et même si cette représentation est fausse, la réalité des partis pris locaux se retrouve prisonnière de représentations reproduites de revues en revues au cours du temps.

Dans un contexte très différent, on retrouve le même type de tension entre revendication d'une spécificité nationale et aspiration à une intégration à la scène globale de l'art dans les ambivalences de la scène chinoise de l'art contemporain, ce dont témoignent les textes de Shiyan Li et Anny Lazarus. Shiyan Li prend pour prétexte la difficulté qu'il y a à traduire l'idée même d'art abstrait en chinois. Cette difficulté se lit dans les différentes tentatives menées par plusieurs générations d'artistes et de théoriciens pour transposer cette notion dans le contexte chinois. La phase la plus récente de cette histoire correspond aux tentatives du critique et théoricien Gao Minglu visant à définir une abstraction spécifiquement chinoise, au travers de l'idée de "Maximalisme ", puis avec l'École de l'intentionnalité. Anny Lazarus reprend pour partie les mêmes questions, mais en se limitant aux discussions les plus contemporaines entourant l'idée selon laquelle il existerait une critique d'art spécifiquement chinoise. Les positions de Gao Minglu, bien que centrales dans les débats contemporains, sont semblet-il remises en cause actuellement; l'idée de spécificité chinoise étant vue comme un avatar de la politique nationaliste du moment. 
Le texte de Katja Gentric change de perspective en partant des pratiques d'un ensemble d'artistes sud-africains. À travers ces pratiques, l'auteure livre une étude du contexte artistique de l'Afrique du Sud post-apartheid, un contexte où de nombreux artistes sont confrontés à des situations d'affirmation identitaire. La question des langues et des traductions est au centre de la pratique des artistes dont il est question, lesquels ne se préoccupent pas tant de confrontations globales que de lignes de fracture beaucoup plus locales.

Jovan Mrvaljevic s'interroge enfin sur l'intégration de la scène de l'art d'Europe de l'Est au sein du système de l'art contemporain globalisé. Comme le constate l'auteur, la situation a complètement changé depuis la chute du Mur de Berlin; cette partie du monde étant de moins en moins considérée comme un "bloc » et de plus en plus comme un ensemble de scènes distinctes, où le rapport à la globalisation se joue différemment entre les artistes. En somme, si la globalisation est présente, c'est davantage en filigrane, en tant que désir de reconnaissance des artistes en Occident.

Un entretien réalisé avec Pedro Pablo Gómez à propos de la « décolonisation " complète ce dossier. Enfin, nous accueillons un portfolio d'œuvres de Fabien Mérelle, ainsi qu'un certain nombre de comptes rendus d'ouvrages et d'expositions.

Jérôme Glicenstein

octobre 2016 